\title{
Progress of Multicolor Single Detector to Detector Array Development For Remote Sensing
}

\author{
M. Nurul Abedin ${ }^{1}$, Tamer F. Refaat ${ }^{2}$, Ishwara Bhat ${ }^{3}$, Yegao Xiao ${ }^{3}$, \\ Sumith Bandra ${ }^{4}$, and Sarath D. Gunapala ${ }^{4}$. \\ ${ }^{1}$ NASA Langley Research Center, Hampton, VA 23681 \\ ${ }^{2}$ Science and Technology Corporation, Hampton, VA 23666 \\ ${ }^{3}$ Rensselaer Polytechnic Institute, Troy, NY 12180 \\ ${ }^{4}$ Jet Propulsion Laboratory, Pasadena, CA 91109
}

\begin{abstract}
Knowledge of the spatial and temporal distribution of atmospheric species such as $\mathrm{CO}_{2}, \mathrm{O}_{3}, \mathrm{H}_{2} \mathrm{O}$, and $\mathrm{CH}_{4}$ is important for understanding the chemistry and physical cycles involving Earth's atmosphere. Although several remote sensing techniques are suitable for such measurements they are considered high cost techniques involving complicated instrumentation. Therefore, simultaneous measurement of atmospheric species using a single remote sensing instrument is significant for minimizing cost, size and complexity. While maintaining the instrument sensitivity and range, quality of multicolor detector, in terms of high quantum efficiency and low noise are vital for these missions. As the first step for developing multicolor focal plan array, the structure of a single element multicolor detector is presented in this paper. The detector consists of three p-n junction layers of $\mathrm{Si}, \mathrm{GaSb}$ and InAs wafer bonded to cover the spectral range UV to $900 \mathrm{~nm}, 800 \mathrm{~nm}$ to $1.7 \mu \mathrm{m}$, and $1.5 \mu \mathrm{m}$ to $3.4 \mu \mathrm{m}$, respectively. Modeling of the absorption coefficient for each material was carried out for optimizing the layers thicknesses for maximum absorption. The resulted quantum efficiency of each layer has been determined except InAs layer. The optical and electrical characterization of each layer structure is reported including dark current and spectral response measurements of Si pin structure and of GaSb and InAs p-n junctions. The effect of the material processing is discussed.
\end{abstract}

Keywords: multi-color, UV-far infrared, $\mathrm{Si}$, InAs, GaSb, atmospheric chemistry, remote sensing

\section{INTRODUCTION}

The major contributions for weather and climate changes are due to temporal and spatial distribution of aerosols, clouds, $\mathrm{CO}_{2}, \mathrm{O}_{3}, \mathrm{H}_{2} \mathrm{O}$, and $\mathrm{CH}_{4}$. Considerable interest exists in the development of multicolor detector, which can be used to detect these species. These species have absorption spectra from UV to IR that can be used to make accurate measurements of their distribution in the troposphere and lower stratosphere. Multicolor detector technology can be utilized to develop the active and passive remote sensing instruments for measurements of these species for eventual application to space. A review of multicolor detector technology was discussed in ref. 1 and the progress of this effort has been discussed in this paper.

Multicolor detector technology is rapidly advancing on a number of exciting applications, such as remote sensing and imaging, military, and medical imaging. HgCdTe and QWIP technology is expanding in single-color to multicolor detectors and recently, QWIP have demonstrated four-color detector arrays ${ }^{2}$. Development of multi-band array will continue and two-band detectors were demonstrated based on $\mathrm{HgCdTe} \mathrm{FPAs}^{3}$. A joint effort among NASA Langley, JPL, and RPI is in place for developing a multicolor FPA with 3-dimensional structures to provide high resolution spectroscopic imaging Fourier-Transform interferometers.

At present, the availability of multicolor detectors is limited to the 3-15 microns spectral range and no multicolor detector technology has been reported spanning the spectral range of the UV-to-Far IR. The advancement of the multicolor detector development has stimulated for detecting column amounts of trace gases in the broad wavelength range. This technology development will be very valuable to the NASA Earth and Space Science Enterprises and 
Planetary Exploration Program, allowing critical measurements with improved accuracy and greatly reduced system complexity, weight, and cost. Using classical optical components such as filters, prisms and gratings to separate the desired wavelengths before they reach the detectors results in complex optical systems composed of heavy components. A simpler system would result by utilizing a single optical system and a detector that responds separately to each wavelength band as shown in Fig. 1 (Band1: detector\#1, Band2: detector\#2, Band3: detector\#3, Band4: detector\#4, and Band5: detector\#5). The multicolor detector approach has the potential for application to several other terrestrial and planetary remote sensing applications.

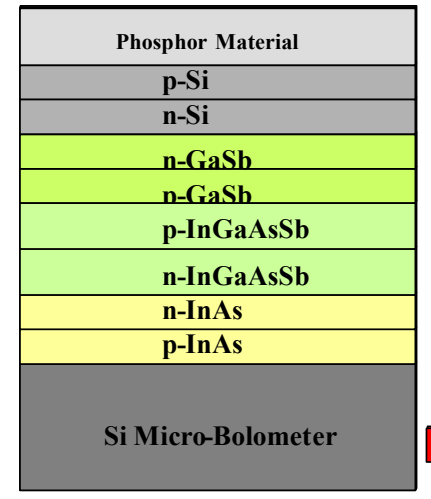

(a)

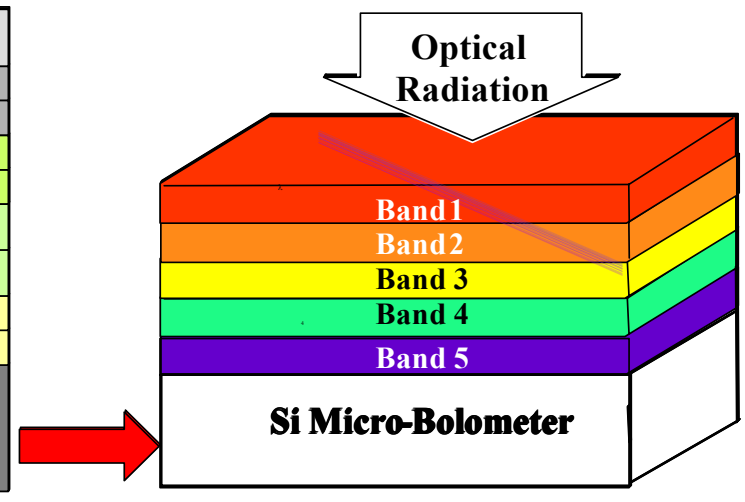

(b)

Fig.1. Schematic of multi-layer bias selectable multi-wavelength IR detector:

(a) Single element layered structures and (b) 3-D Focal Plane Array Detector structures.

In order to achieve the wavelengths either less than 1-micron or greater than 15-micron, one can shorten the operating wavelengths by using phosphor coating on Si material and extend the operating wavelengths by using Simicrobolometer. In this study, there are significant challenges in shortening the wavelength to ultraviolet range using $\mathrm{Si}$ by applying phosphor material on it to improve the UV region and also in extending the wavelength of operation of detectors beyond 15 microns using Si-microbolometer due to hybridization. Quaternary layers of GaInAsSb have been grown by metal organic chemical vapor deposition (MOCVD) with good optical and electrical properties. These layers are lattice-matched to $\mathrm{GaSb}$ or InAs substrates with cutoff wavelengths for absorption tailored from 1.7- to 2.5- $\mu \mathrm{m}$. Epitaxial growth on a lattice-matched substrate is crucial in reducing unwanted defects that could lead to higher noise in the detector. There are at present few materials discussed above with varying band gaps that cover the ultraviolet to far IR wavelength band with lattice-matched conditions.

Therefore, a multicolor detector array, sensitive in broadband range with different bands covering the 0.4- $\mu \mathrm{m}$ to far-IR wavelength range, is proposed. To achieve this, it is anticipated that silicon ( $\mathrm{Si})$, gallium antimonite $(\mathrm{GaSb})$, indium arsenide (InAs), III-V semiconductor ternary and quaternary alloy-based materials, vertically integrated, heterostructure p-i-n diodes grown via metal-organic vapor phase epitaxy (MOVPE) and also wafer bonding techniques can be utilized. Adding up to four-to-five materials expands the flexibility of the semiconductor material system so that photoexcitation band gaps and lattice parameters can be adjusted independently, allowing us to find a lattice-matched substrate on which to grow these multicolor detector stacks monolithically, or lattice-mismatched detector stacks using wafer bonding technology.

In this paper, we have discussed modeling and characterization of $\mathrm{Si}, \mathrm{GaSb}$, and InAs detectors. Modeling of the absorption coefficient of each material has been carried out for optimizing the layer thicknesses for optimum absorption and high quantum efficiency of each layer. The optical and electrical characterization of each of the layer structure has been presented including spectral response and dark current measurements of $\mathrm{Si}$ pin structure and of $\mathrm{GaSb}$ and InAs p-n junctions. Responsivity measurements are performed as a function of incident wavelengths and dark currents are measured with applied bias voltages. 


\section{MULTICOLOR SINGLE ELEMENT DETECTOR STRUCTURES}

The integration of silicon technology with antimonide based material system for the simultaneous detection of multi-wavelengths in the visible and the infrared (IR) is attempted. GaSb, AlSb and InAs are closely lattice-matched, but have widely different bandgaps. GaSb has a bandgap of $0.72 \mathrm{eV}$, AlSb has an indirect band gap of $1.63 \mathrm{eV}$ and InAs has a bandgap of $0.356 \mathrm{eV}$. The lattice constant and the band gaps of these materials and some other related materials are shown in Table 1.

Table 1. Lattice constant and bandgaps of some binary compounds.

\begin{tabular}{|l|l|l|}
\hline Material & band gap (eV) & lattice constant $(\mathbf{\AA})$ \\
\hline \hline $\mathrm{GaSb}$ & 0.72 & 6.0959 \\
\hline $\mathrm{AlSb}$ & $1.63(\mathrm{I})$ & 6.136 \\
\hline $\mathrm{InSb}$ & 0.17 & 6.479 \\
\hline $\mathrm{GaAs}$ & 1.43 & 5.6532 \\
\hline $\mathrm{AlAs}$ & $2.165(\mathrm{I})$ & 5.6622 \\
\hline $\mathrm{InAs}$ & 0.356 & 6.0583 \\
\hline
\end{tabular}

As shown, the lattice constants of these materials are not perfectly matched, and hence we will still get high density of dislocations when one material is grown on the other. However, one can grow ternary and quaternary layers lattice-matched to one of the binaries, and the band gap of the quaternary layers can be tuned to any desired wavelength, still maintaining the lattice-matched condition. This allows great flexibility in device design, but the material growth will be more complicated. However, recent advances in MOVPE growth technique can allow reproducible growth of such quaternary layer possible. Growth of high quality quaternary layers was demonstrated very recently for use in thermophotovoltaic systems. ${ }^{4,5}$

The multi-wavelength device structure in the UV-to-IR band is shown in Fig. 1. An n-type GaSb substrate is chosen on which a p-on-n InAs device structure is grown, followed by an n-on-p second IR detector in a quaternary layer of InGaAsSb. The band gap of the quaternary layer can be changed from $0.29 \mathrm{eV}$ to $0.35 \mathrm{eV}$ or from $0.7 \mathrm{eV}$ to $0.5 \mathrm{eV}$ (wavelength cut-off from 4.3- to 3.5- $\mu \mathrm{m}$ or from 1.7- to $2.5-\mu \mathrm{m}$ ), still maintaining the lattice matching with the substrate.

One important point to remember in the above structure is the band alignment. InAs-GaInAsSb has type-II band alignment. In the above configuration, i.e., n-p-P-N configuration, the minority carrier electrons in the P-GaInAsSb will not be collected by the lower band gap junction. However, the electrons from the lower band gap p-type layer can be collected by the common junction, and hence there will be a small amount of cross talk. The independent bias for the two junctions will alleviate this problem, and in addition, the p-type lower band gap material thickness should be kept to a minimum to reduce this cross talk further.

We propose to use wafer-bonding approach to overcome the lattice mismatch problem present in ternary layers. This allows us to optimize the detectors for each region of operation to achieve high quantum efficiency. The proposed multicolor detector is based on the integration of Si-microbolometer with the Si and the III-V compound-based IR detectors to obtain the wavelengths of interest. The IR detector structures used are grown on the GaSb substrate using MOVPE. The composite single detector layered structures are shown in Fig. 1a.

Fig. $1 \mathrm{~b}$ shows the 3-D detector sketch with input radiation from the optical system impinging the detector stack. Light of the appropriate wavelengths of interest is absorbed in the first detector in this "sandwich", while longer wavelengths are not absorbed. Longer wavelengths are passed into the second detector and this second set of wavelengths is absorbed in the second detector. The desired absorption wavelengths in each detector can be tailored for each atmospheric or geophysical sounding application, making for a very efficient instrument, as unnecessary spectral lines or spectral bands are not processed. 


\section{MODELING OF ABSORPTION COEFFICIENT AND QUANTUM EFFICIENCY OF EACH LAYER STRUCTURE}

The multi-color detector development effort based on numerical calculations of absorption coefficient and quantum efficiency has been discussed. This effort includes evaluation of the absorption spectrum of the basic $\mathrm{Si}, \mathrm{GaSb}$, and InAs materials as a function of incident radiation. Experimental absorption spectra were obtained from different groups through literature search to compare with numerical values. The absorption coefficients were calculated using dielectric function and is given by ${ }^{6}$

$$
\varepsilon(\omega)=\varepsilon_{1}(\omega)+j \cdot \varepsilon_{2}(\omega)
$$

The real $\left(\varepsilon_{1}\right)$ and imaginary $\left(\varepsilon_{2}\right)$ parts of the dielectric function are related by the Kramers-Kronig relations as discussed in ref. 6 :

$$
\begin{aligned}
& \varepsilon_{1}(\omega)=1+\frac{2}{\pi} \int_{0}^{\infty} \frac{\omega^{\prime} \cdot \varepsilon_{2}\left(\omega^{\prime}\right)}{\left(\omega^{\prime}\right)^{2}-\omega^{2}} \cdot \mathrm{d} \omega^{\prime} \\
& \varepsilon_{2}(\omega)=-\frac{2}{\pi} \int_{0}^{\infty} \frac{\omega^{\prime} \cdot \varepsilon_{1}\left(\omega^{\prime}\right)}{\left(\omega^{\prime}\right)^{2}-\omega^{2}} \cdot \mathrm{d} \omega^{\prime}
\end{aligned}
$$

The optical absorption coefficient $\alpha(\omega)$ is related to the dielectric function by the relation ${ }^{6}$

$$
\alpha(\omega)=\frac{4 \pi}{\lambda} \cdot \sqrt{\frac{\sqrt{\varepsilon_{1}(\omega)^{2}+\varepsilon_{2}(\omega)^{2}}-\varepsilon_{1}(\omega)}{2}}
$$

The absorption coefficients, $\alpha=\sim 2 \times 10^{4} \mathrm{~cm}^{-1}$ at $\lambda=0.6-\mu \mathrm{m}$ wavelength for $\mathrm{Si}, \alpha=\sim 1 \times 10^{4} \mathrm{~cm}^{-1}$ at $1.6-\mu \mathrm{m}$ wavelength for $\mathrm{GaSb}$, and $\alpha=3 \times 10^{3} \mathrm{~cm}^{-1}$ at 3.3- $\mu \mathrm{m}$ wavelength for InAs, are noted (See Fig. 2a). The photon flux absorbed by each layer is shown in Fig. 2b.

In addition, quantum efficiencies of $\mathrm{Si}$ and of $\mathrm{GaSb} \mathrm{n}+\mathrm{p}$ device structures are determined using PC1D simulation software tool. The device structure is shown in Fig. 3, and some of its important parameters are listed in Table 2 for $\mathrm{Si}$ and Table 3 for GaSb. The simulation depends on the information associated with structure, material parameters, and physical coefficients. The structure can be simulated either as a two-region device (one $\mathrm{n}+$ and another p-type) or as a one-region device with p-type background and $n+$ diffusion. Both approaches provide similar results, and here, some of the results from the latter approach are presented.

Fig. 4 shows quantum efficiency (QE) vs wavelength with respect to front reflection coefficients. The highest $\mathrm{QE}$ is achieved at wavelength around $600 \mathrm{~nm}$, which is the visible part of Si material absorption spectrum. Antireflection coating improves QE, but, on the other hand, QE also can be affected by the doping density and length of the $\mathrm{n}+$ region. Figs. 5a and 5b show the QE with different $\mathrm{n}+$ doping density (see Fig. 5a) and QE with different $\mathrm{n}+$ region length (see Fig. 5b), respectively. It can be seen from the figures that, when the $\mathrm{n}+$ doping density is very high $\left(>10^{19}\right.$ $\mathrm{cm}^{-3}$ ), or when the $\mathrm{n}+$ region length is high (beyond $3 \mu \mathrm{m}$ ), the QE would be significantly reduced. This is probably because of the large recombination rate when $\mathrm{n}+$-doping density is high or when the $\mathrm{n}+$ region length is large.

Fig. 6 shows quantum efficiency (QE) vs wavelength with respect to front reflection coefficients for GaSb. The highest QE is achieved at wavelength around $1760 \mathrm{~nm}$, close to the band gap of GaSb material. Figs. 7a and 7b show the QE with different $n+$ doping density (see Fig. 7a) and QE with different $n+$ region length (see Fig. 7b), respectively. It can be seen from the figures that, when the $\mathrm{n}+$ doping density is very high (e.g., $\sim 10^{19} \mathrm{~cm}^{-3}$ ), or when the $\mathrm{n}+$ region length is high (beyond $3 \mu \mathrm{m}$ ), the QE would be significantly reduced. Again, this is due to the large recombination rate when $\mathrm{n}+$ doping density is high or when the $\mathrm{n}+$ region length is large, as seen from Figs. $8 \mathrm{a}$ and $8 \mathrm{~b}$, respectively. At shorter wavelength, most of the light is absorbed closer to the surface, and hence high recombination rate at the surface reduces the photoresponse considerably. Hence thinner $\mathrm{n}+$ region with low surface recombination velocity and high diffusion length (low $\mathrm{n}+$ doping) is essential for good device. 


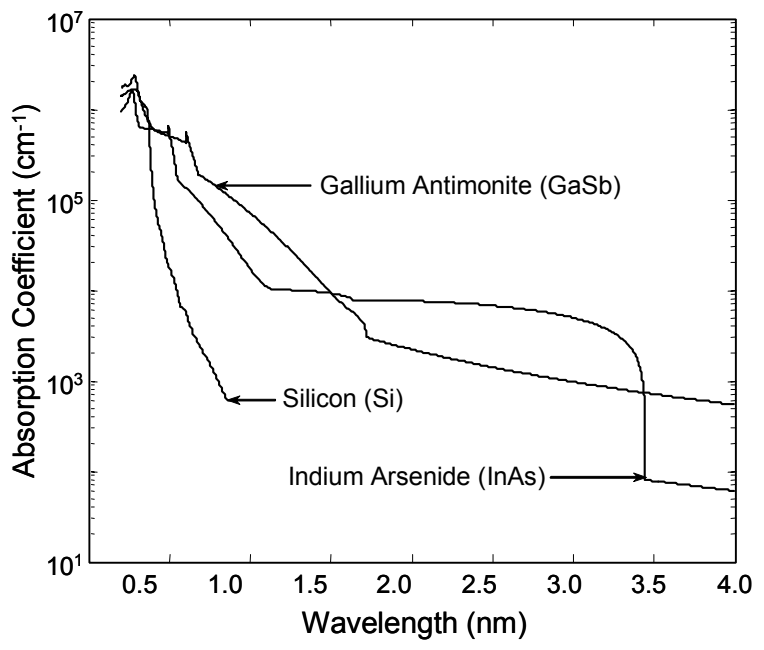

(a)

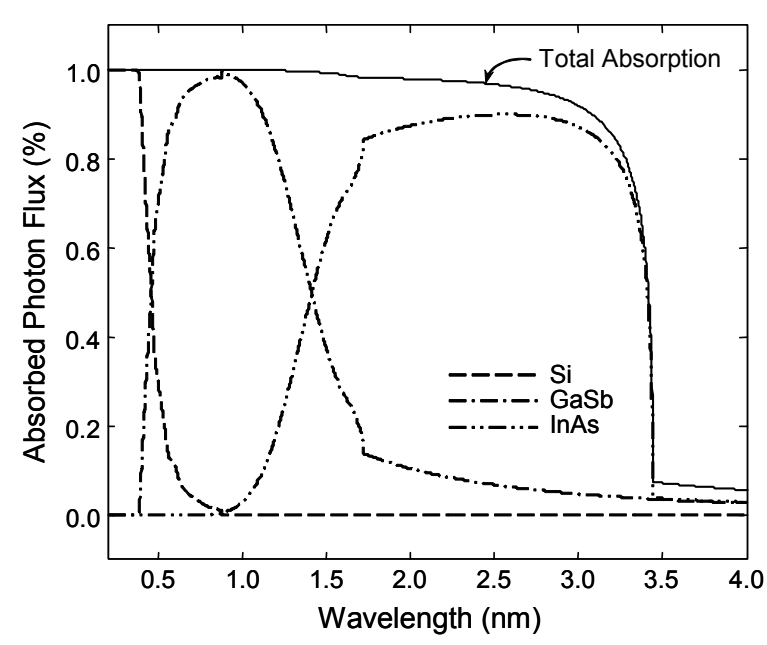

(b)

Fig. 2. Calculated Absorption coefficients (a) and absorbed photon flux (b) of the basic $\mathrm{Si}, \mathrm{GaSb}$, and InAs materials as a function of incident radiation.

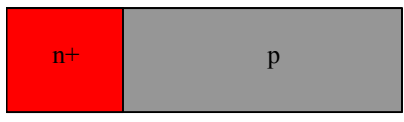

Fig. 3. Schematic of a $\mathrm{Si} \mathrm{n}+\mathrm{p}$ diode structure.

Table 2. Structure and material parameters, and physical coefficients for $\mathrm{Si} \mathrm{n}+\mathrm{p}$ device for simulation.

\begin{tabular}{|l|l|l|l|l|}
\hline \hline Length/n+ & Total $/ \mathrm{n}+\mathrm{p}$ & Ext refl & Band gap & Intrinsic concentr (300k) \\
\hline $2 \mu \mathrm{m}$ & $12 \mu \mathrm{m}$ & $5 \%$ & $1.124 \mathrm{eV}$ & $1.0 \times 10^{10} \mathrm{~cm}^{-3}$ \\
\hline \hline Backkgrd $/ \mathrm{p}$ & Doping/n+ & Temp & Elect affinity & Ref index \\
\hline $1 \times 10^{16} \mathrm{~cm}^{-3}$ & $1 \times 10^{18} \mathrm{~cm}^{-3}$ & $300 \mathrm{~K}$ & $4.05 \mathrm{eV}$ & Ext file \\
\hline \hline Absorp Coeff & Free carrier absorp & Nc/Nv ratio & Relat dielectr & Recombination \\
\hline Ext file & Enabled & 1.06 & 11.9 & Internal model \\
\hline \hline
\end{tabular}

Table 3. Structure and material parameters and physical coefficients for GaSb $\mathrm{n}+\mathrm{p}$ device for simulation ${ }^{8-10}$.

\begin{tabular}{|l|l|l|l|l|}
\hline \hline Length/n+ & Total/n+ $+\mathrm{p}$ & Ext refl & Band gap & Intrinsic concentr (300k) \\
\hline $3 \mu \mathrm{m}$ & $12 \mu \mathrm{m}$ & $5 \%$ & $0.726 \mathrm{eV}$ & $1.52 \times 10^{12} \mathrm{~cm}^{-3}$ \\
\hline Backkgrd $/ \mathrm{p}$ & Doping/n+ & Temp & Elect affinity & Ref index \\
\hline $5 \times 10^{16} \mathrm{~cm}^{-3}$ & $2.5 \times 10^{18} \mathrm{~cm}^{-3}$ & $300 \mathrm{~K}$ & $4.06 \mathrm{eV}$ & Ext file \\
\hline \hline Absorp Coeff & Free carrier absorp & $\mathrm{Nc} / \mathrm{NV}$ ratio & Relat dielectr & Recombination \\
\hline Ext file & Enabled & 0.0114 & 15.7 & Internal model \\
\hline \hline
\end{tabular}




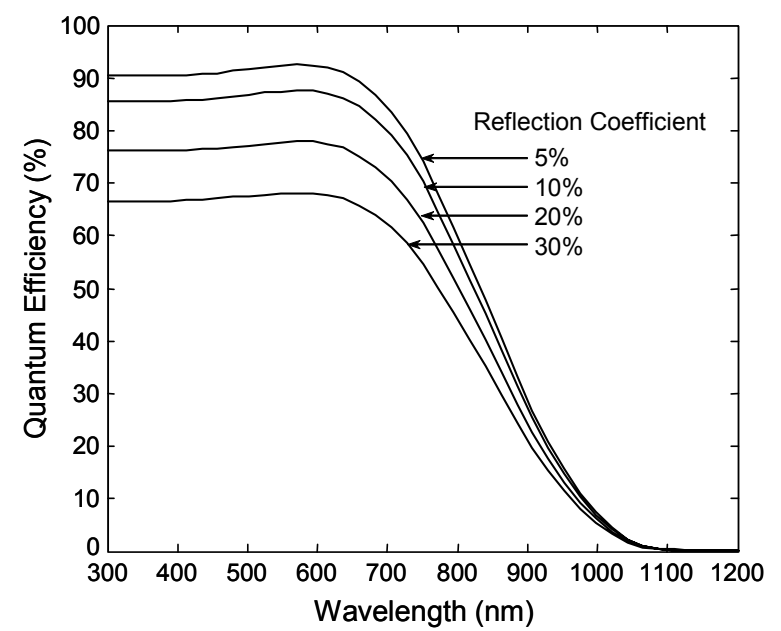

Fig. 4. QE vs wavelength of Si at different front reflection coefficient values.

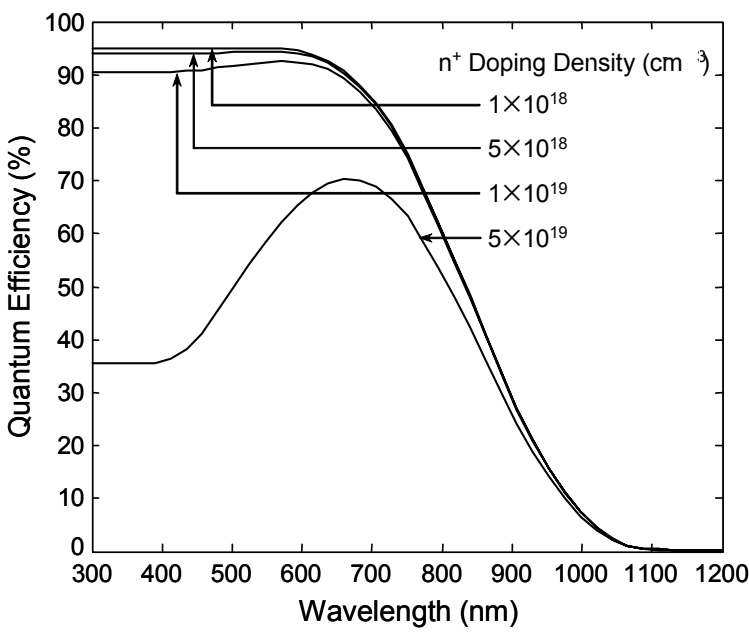

(a)

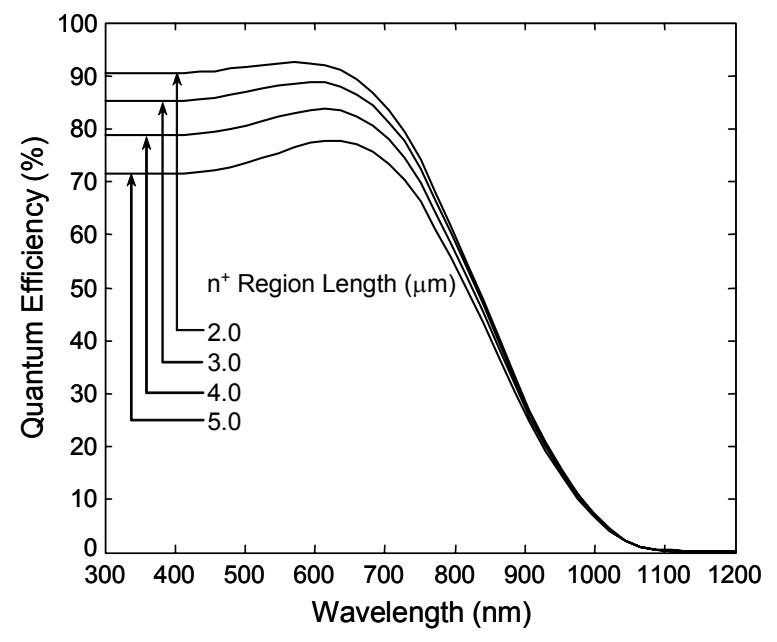

(b)

Fig. 5. QE vs wavelength of Si with different $\mathrm{n}+$ doping density values (a), and with different $\mathrm{n}+$ region lengths (b).

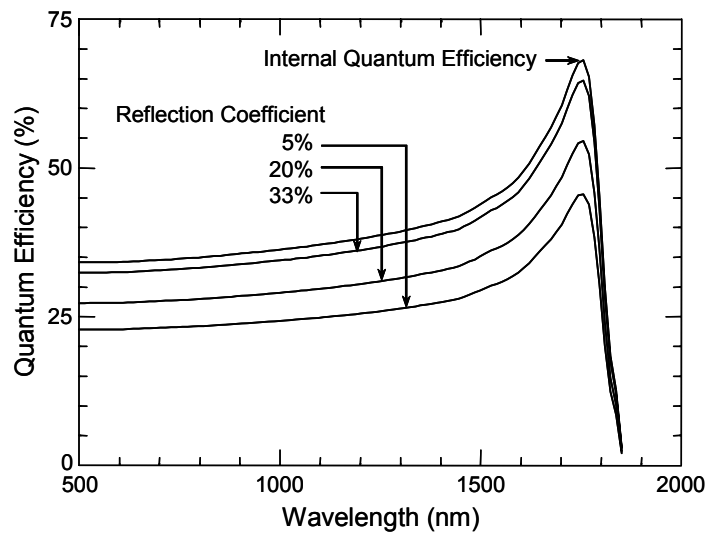

Fig. 6. QE vs. wavelength of GaSb at different front reflection coefficient values. 

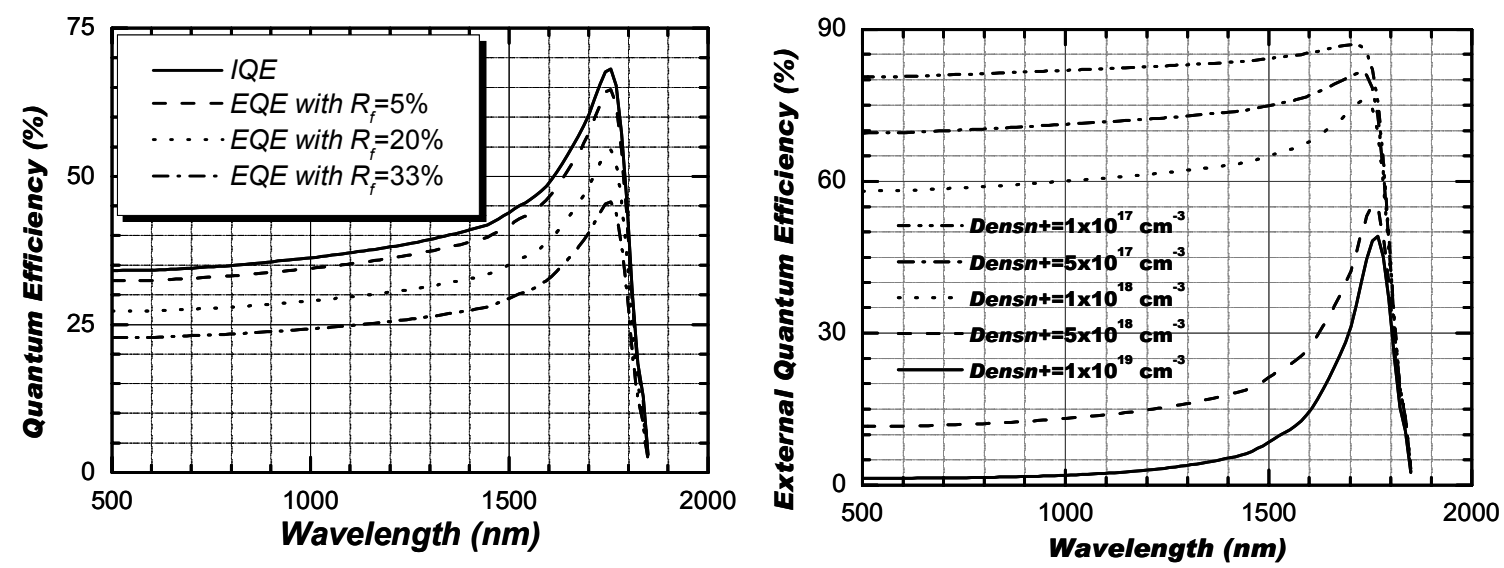

Fig. 7. QE vs. wavelength of GaSb with different $n+$ doping density values (a), and with different $n+$ region lengths (b).

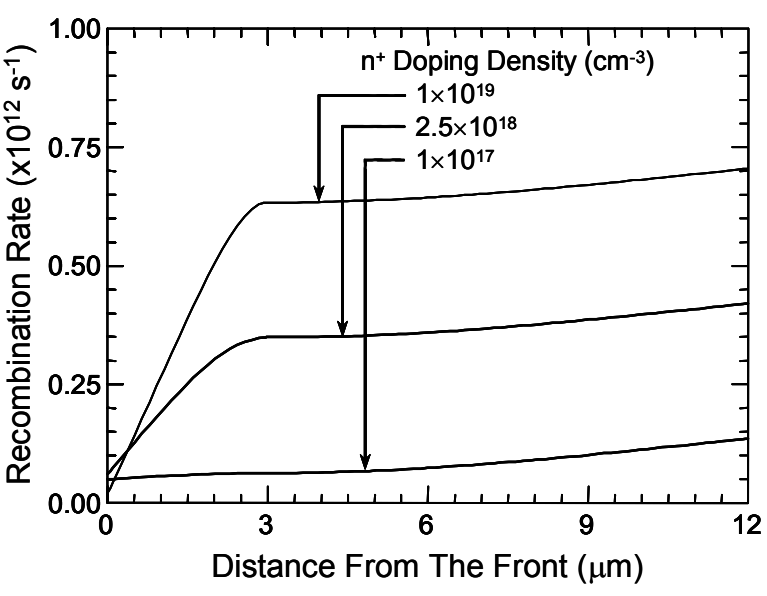

(a)

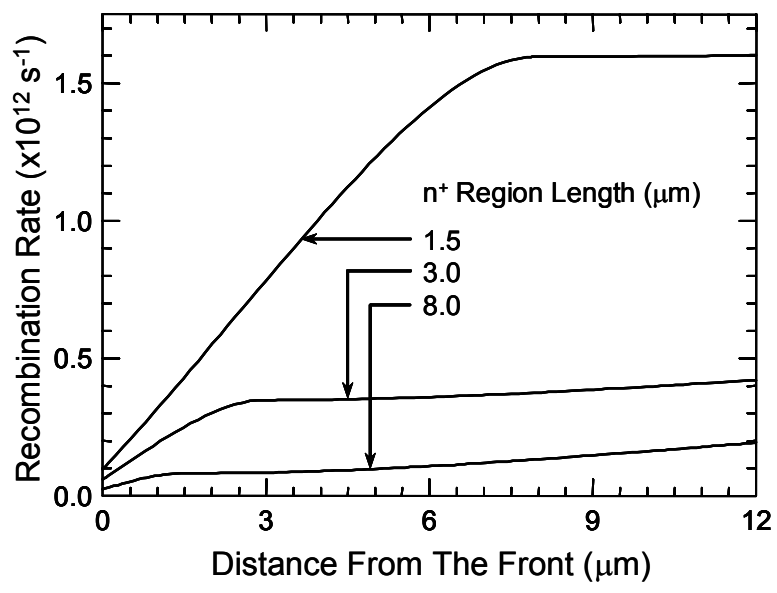

(b)

Fig. 8. Recombination rate with different $\mathrm{n}+$ doping density values (a), and with different $\mathrm{n}+$ region lengths $(\mathrm{b})$ of $\mathrm{GaSb}$.

\section{EXPERIMENTAL RESULTS AND DISCUSSIONS}

$\mathrm{Si}, \mathrm{GaSb}$, and InAs detectors were acquired from Perkin Elmer, Rensselaer Polytechnic Institute, and Judson Technologies; and characterized to obtain the dark current and spectral response. The current vs. voltage (I-V) characteristics were obtained for these detectors using a HP modular DC source/monitor. Fig. 9 shows the dark current variation with the reverse bias voltage at $20{ }^{\circ} \mathrm{C}$ for the detector samples. The dark current was obtained in dark conditions by applying the bias voltage through the p-side while the $n$-side was connected to ground. Data was acquired at $10 \mathrm{mV}$ bias step using 10 averages for the $\mathrm{GaSb}$ and InAs detectors, while $75 \mathrm{mV}$ step was used for the Si detector. The estimated maximum standard deviation is $5 \%$ for the shown data. At a fixed temperature the dark current increases with bias voltage. The lowest dark current was observed for the Si pin device structure as expected. Other two tested $\mathrm{GaSb}$ and InAs devices showed high dark current compared to the theoretical values, as it is determined from the current vs. voltage characteristics at different bias voltages with fixed room temperature. This supports the need for improvement of $\mathrm{GaSb}$ and InAs device processing, especially surface passivation, which will provide low dark current and eventually will be utilized for multicolor detector development. 


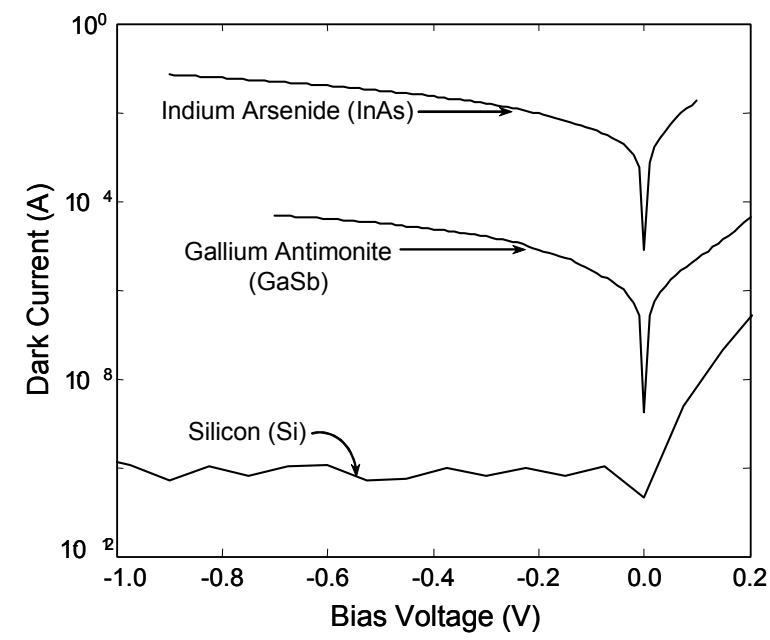

Fig. 9. Dark current measurement of Si (Perkin Elmer; C30807E), GaSb (RPI) and InAs (Judson Technologies; J12-18C-R01M) detector samples, obtained at $20{ }^{\circ} \mathrm{C}$.

The spectral responsivity of several detector samples was at $20^{\circ} \mathrm{C}$ and $0 \mathrm{~V}$ bias voltage with $20 \mathrm{~nm}$ resolution. The detector samples comprise of a Si pin detector obtained from Perkin Elmer (C30807E) with 400 to $1100 \mathrm{~nm}$ sensitivity, GaSb pn detector fabricated at RPI with 800 to $1700 \mathrm{~nm}$ sensitivity and InAs pn detector obtained from Judson Technologies (J12-18C-R01M) with 1300 to $3800 \mathrm{~nm}$ sensitivity. All detectors have $1 \mathrm{~mm}$ sensitive area diameter. The spectral response was obtained using the substitution method with two calibrated reference $\mathrm{Si}$ and $\mathrm{PbS}$ detectors, for the spectral calibration between 250 to $1100 \mathrm{~nm}$ and 1000 to $3200 \mathrm{~nm}$, respectively. A standard current controlled quartz halogen lamp was used as the radiation source with its output modulated using a chopper. A monochromator is used to separate the radiation into its spectral components, with dispersion filters at its output. The detector output current is converted into a voltage signal using a preamplifier (Stanford Research Systems; SR570), the output of which is applied to a lock-in amplifier (Optronic Laboratories; 750-C). A personal computer controls the setup operation and stores the acquired data. Figure 10 shows the spectral responsivity of the Si pin diode in the 300 to 1100 $\mathrm{nm}, \mathrm{GaSb}$ pn photodiode in the 1000 to $1800 \mathrm{~nm}$, and InAs pn photodiode in the 1200 to $3200 \mathrm{~nm}$ spectral range. The peak responsivities of these detectors are at 0.85-, 1.55- and 3.2-micron at room temperature for $\mathrm{Si}, \mathrm{GaSb}$ and InAs, respectively.

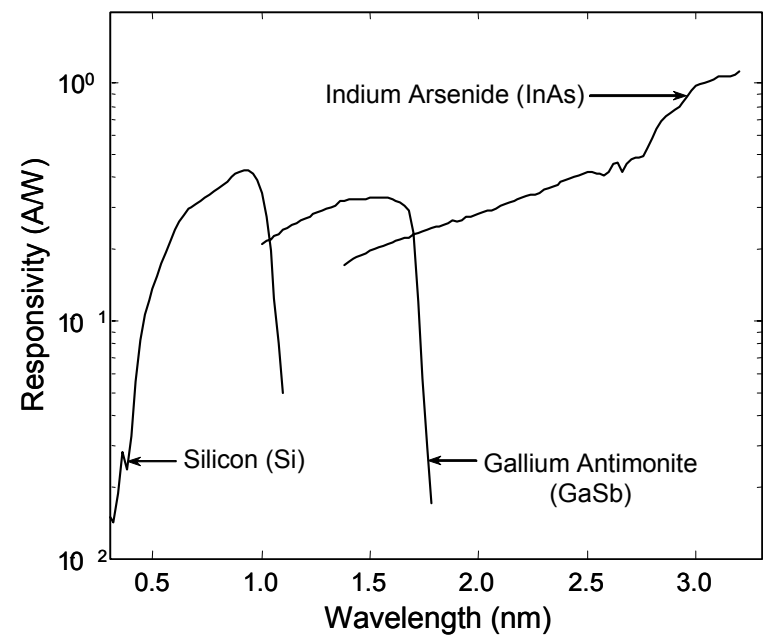

Fig. 10. Spectral response of Si (Perkin Elmer; C30807E), GaSb (RPI) and InAs (Judson Technologies; J12-18C-R01M) detector samples, obtained at $0 \mathrm{~V}$ bias and $20{ }^{\circ} \mathrm{C}$. 


\section{CONCLUSION}

We have demonstrated the feasibility of this multicolor detector development effort by acquiring and characterizing $\mathrm{Si}, \mathrm{GaSb}$, and InAs single element detectors. Numerical calculations have been performed of these devices to obtain the absorption coefficients and photon absorption flux in each layer and also to optimize the quantum efficiency of those material systems. Current vs. voltage and spectral response measurements were carried out at Langley's Detector Characterization Laboratory. Dark current and responsivity were determined at bias voltages and incident radiation with fixed temperature. Experimental results obtained from these devices are promising for future development of multicolor detector using $\mathrm{Si}, \mathrm{GaSb}$, InAs, InGaAsSb, and Si-microbolometer detectors in the spectral range UV-to-far IR.

\section{ACKNOWLEDGEMENT}

This effort is part of the Breakthrough Atmospheric Sensing funded by NASA's Earth Science Technology Office and the Enabling Concepts and Technology Program within NASA's Aerospace Technology Enterprise. The authors would like to thank Frank Peri for his support.

\section{REFERENCES}

1. M. Nurul Abedin, Tamer F. Refaat, Joseph M. Zawodny, Stephen P. Sandford, Upendra N. Singh, Sumith V. Bandara, Sarath D. Gunapala, Ishwara Bhat, and N.P. Barnes, "Multicolor Focal Plane Array Detector: A Review", Proc. SPIE Vol. 5152, Infrared Spaceborne Remote Sensing XI, pp. 279 - 288 (2003).

2. S.V. Bandara, S. D. Gunapala, J. K. Liu, S. B. Rafol, D. Z. Ting, J. M. Mumolo, R. W. Chuang, T. Q. Trinh, J. H. Liu, K. K. Choi, M. Jhabvala, J. M. Fastenau, W. K. Liu "Four-band Quantum Well Infrared Photodetector Array", Infrared Physics and Technology 44, pp. 369-375 (2003).

3. R.D. Rajavel, P.D. Brewer, D.M. Jamba, J.E. Jensen, C. LeBeau, G.L. Olson, J.A. Roth, W.S. Williamson, J.W. Bangs, P. Goetz, J.L. Johnson, E.A. Patten, and J.A. Wilson, "Status of HgCdTe-MBE technology for producing dual-band infrared detectors", J. Crystal Growth 214/215, 1100-1105 (2000).

4. C. Hitchcock, R. Gutmann, J. Borrego, I. Bhat, and G. Charache, "Antimonide based devices for thermophotovoltaic applications", IEEE Transactions on Electron Devices, 46, 2154, (1999).

5. C.A. Wang, H.K. Choi, S.L. Ransom, "High-quantum-efficiency $0.5 \mathrm{eV} \mathrm{GaInAsSb/GaSb}$ thermophotovoltaic devices" Applied Physics Letters, Vol. 75, 1305 (1999).

6. S. Adachi, "Physical Properties of III-V Semiconductor Compounds: InP, InAs, GaAs, GaP, InGaAs, and InGaAsP", John Wiley \& Sons, Inc., p. 135 (1992).

7. PC1D Version 5.9 for Windows, Centre for Photovoltaic Engineering UNSW, Univ. of New South Wales (2003).

8. S. Wang, "Fundamentals of semiconductor theory and device physics", Prentice Hall, (1989).

9. A. G. Milnes and A. Y. Polyakov, "Gallium antimony device related properties", Solid State Electronics, 36(6), 803-818 (1993).

10. H. Ehsani, I. Bhat, C. Hitchcock, R. J. Gutmann, G. Charache, and M. Freeman, "p-type and n-type doping in $\mathrm{GaSb}$ and $\mathrm{Ga}_{0.8} \mathrm{In}_{0.2} \mathrm{Sb}$ layers grown by metalorganic vapor phase epitaxy", J. Crystal Growth, 195, 385-390 (1998). 